\title{
The Regulation of Glutamine Metabolism in Candida utilis: the Role of Glutamine in the Control of Glutamine Synthetase
}

\author{
By A. R. FERGUSON AND A. P. SIMS* \\ School of Biological Sciences, University of East Anglia, Norwich, NOR 88C
}

(Received 7 March 1973 ; revised 12 July 1973)

\section{SUMMAR Y}

In Candida utilis, the amount of glutamine synthetase is finely attuned to the availability of ammonia in the culture medium, and any change in ammonia availability results in a compensatory adjustment to enzyme level. In yeast growing with different sources of nitrogen there was a good inverse correlation between the rate of enzyme synthesis and the size of the cell pool of glutamine: glutamine and not ammonia appeared to act as a co-repressor of the formation of glutamine synthetase.

Sudden changes in ammonia availability or carbon supply could lead to the inactivation of glutamine synthetase. This was shown to be associated, under some conditions, with a marked increase in the pool of glutamine and it is possible that it was brought about by sudden changes in the relative cell concentrations of glutamine and glutamate. However, the process is clearly more complex than this and catabolism of carbon substrates is also involved.

The specific activity of the NAD-specific (deaminating) glutamate dehydrogenase also varied with the availability of ammonia, but neither it nor glutamine synthetase was subject to parallel regulation.

\section{INTRODUCTION}

In the food yeast Candida utilis, the rate of glutamine synthesis has been shown to be determined primarily by the amount of glutamine synthetase present, and full expression of enzyme activity appears to be limited only by the availability of substrates, particularly that of ammonia (Sims \& Ferguson, 1972). Studies with partially purified enzyme preparations have revealed that neither glutamine itself nor the possible end-products of its metabolism, singly or in combination, cause any significant inhibition of synthetase activity. Moreover, physiological experiments have confirmed that feedback inhibition cannot play an important role in the regulation of this enzyme (Sims \& Ferguson, 1974).

The observation that changes in enzyme amount are required to compensate for changes in substrate availability posed the question as to how such precise and flexible control of enzyme level is achieved. In this paper we report that glutamine itself is probably the co-repressor of enzyme synthesis and that changes in the relative sizes of the soluble pools of glutamate and glutamine are indirectly involved in the bringing about of rapid adjustments to enzyme level.

\footnotetext{
* All communications should be addressed to A.P.S.
} 


\section{METHODS}

Organism and growth conditions. The strain of Candida utilis used was NCYC 737. The yeast was grown in batch culture in the synthetic medium described by Ferguson \& Sims (I97I), at $27^{\circ} \mathrm{C}$ in a Gallenkamp orbital shaker. In some experiments a 21 turbidostat was used. The level of residual nitrogen in the culture medium (approx. $5 \mu \mathrm{g} \mathrm{N} / \mathrm{ml}$ ) was controlled by approriate adjustment of the cell mass of the culture (approx. $0.7 \mathrm{mg}$ dry wt yeast $/ \mathrm{ml}$ of culture).

Transfer of yeast to different media. Yeast could be transferred rapidly from one medium to another, without disturbing exponential growth, by filtering the culture (up to $500 \mathrm{ml}$ ) on $12 \mathrm{~cm}$ circles of glass-fibre paper (Whatman GFA), washing the pad of yeast with temperature-equilibrated medium, and then transferring it to the new medium. The yeast was dispersed by vigorous agitation and the glass-fibre paper debris was removed by filtering the culture through fine nylon mesh.

In some experiments, it was essential to remove any contaminating traces of ammonia or other nitrogenous compounds from the medium to be used. A sample of exponentiallygrowing cells (about $15 \mathrm{mg}$ dry wt/l) was therefore added to nitrogen-deficient medium and incubated for $30 \mathrm{~min}$ at $27^{\circ} \mathrm{C}$. The yeast was then removed by vacuum filtration and the required nitrogen source was added to the medium.

Preparation of crude yeast extracts and assay of enzyme activities. Crude extracts were prepared and the following enzymes were assayed as described in Ferguson \& Sims (I97I): NAD-specific glutamate dehydrogenase [L-glutamate: NAD oxidoreductase (deaminating), EC.I .4.I.2]; NADP-specific glutamate dehydrogenase [L-glutamate: NADP oxidoreductase (deaminating), EC.I.4.I.4]; and glutamine synthetase [L-glutamate: ammonia ligase (ADP), EC.6.3.I.2] (transferase assay).

Glutamine synthetase activity was also measured by using the synthetase assay. The assay mix contained: adenosine triphosphate, I $2 \mu \mathrm{mol}$; glutamate, $6 \mathrm{I} \mu \mathrm{mol}$; hydroxylamine, I6 $6 \mathrm{~mol}$; magnesium sulphate, I60 $\mu \mathrm{mol}$; all in imidazole buffer, $0.05 \mathrm{M}, \mathrm{pH} 7 \cdot \mathrm{I}$, containing $5 \times 1 \mathrm{IO}^{-4} \mathrm{M}$-EDTA in a final volume of $2.0 \mathrm{ml}$.

In some experiments glutamine synthetase was measured directly in yeast cells that had been frozen in liquid nitrogen and thereby rendered permeable to substrates. A sample of yeast culture (normally between 0.5 and $2.0 \mathrm{mg}$ dry wt) was filtered onto a $2.5 \mathrm{~cm}$ circle of Whatman GFA, frozen in freshly dispensed liquid nitrogen, and stored at - I $8{ }^{\circ} \mathrm{C}$. Glutamine synthetase activity was stable for at least one week.

Reproducible results were obtained with both the transferase and synthetase assays (normally about $\pm 4 \%$ ). The relationship between enzyme activity and quantity of yeast suspension taken was linear, reaction rates were zero order for at least $15 \mathrm{~min}$, and the measured affinities of glutamine synthetase for its various substrates were similar to those obtained with partially-purified enzyme preparations.

The freezing of yeast in liquid nitrogen also allowed the satisfactory assay of other enzymes such as $\beta$-galactosidase, $\alpha$-glucosidase and nitrate reductase (A. P. Sims and A. R. Ferguson, unpublished).

Measurement of nitrogenous compounds in medium. Measurements were carried out on samples of medium after yeast had been removed by vacuum filtration through Whatman GFA paper. Ammonia was determined by the method of Weatherburn (1967), and glutamate as described below.

Preparation of soluble extracts for amino acid analysis. Yeast $(20 \mathrm{ml}$ of culture) was harvested in the exponential phase of growth by vacuum filtration on $2.5 \mathrm{~cm}$ circles of Whatman GFA held in a small Buchner funnel. The pad was washed $(5 \mathrm{ml})$ with chilled 
$0.15 \mathrm{M}-\mathrm{NaCl}$ and extracted with 4 portions $(3 \mathrm{ml})$ of $60 \%(\mathrm{v} / \mathrm{v})$ ethanol. The extracts were stored in a deep-freeze and were taken to dryness on a rotary film evaporator $\left(30{ }^{\circ} \mathrm{C}\right)$ just before analysis of their amino-acid content.

The estimation of glutamate and glutamine. Glutamate was separated from glutamine and other neutral amino acids on columns $(6 \times \mathrm{I} \mathrm{cm})$ of Dowex I $(8 \%$ crosslink, dry mesh 200 to 400 ) (acetate form) by a modification of the method of Hirs, Moore \& Stein (I954). An almost quantitative recovery of glutamate (approx. $97 \%$ ) was achieved by pre-treating the resin (chloride form) with a large excess of $\mathrm{Na}_{2} \mathrm{CO}_{3}$ and then converting it to the acetate form with an excess of $2 \mathrm{M}$-acetic acid. The pad extracts (taken into $2 \mathrm{ml}$ water) were applied to the resin columns, which were then washed first with $10 \mathrm{ml}$ water to rid them of neutral and basic amino acids, then with $15 \mathrm{ml}$ of $0.2 \mathrm{M}$-acetic acid. The first $5 \mathrm{ml}$ of the eluate was discarded and the remainder contained the glutamate free of both tyrosine and aspartate.

The water wash from the Dowex I column was incubated overnight at room temperature with 0.2 units of glutaminase (Sigma Type IV, Sigma London Chemical Co., London) and the glutamate resulting from the enzymatic hydrolysis of glutamine was recovered as above. The amino acids were determined with ninhydrin by a method essentially similar to that of Yemm \& Cocking (1955) except that composition of the ninhydrin reagent was: ninhydrin, $\mathrm{I} \cdot \mathrm{I} 5 \mathrm{~g}$; ascorbic acid, $40 \mathrm{mg}$; water, $4.0 \mathrm{ml}$; and 2-methoxy-ethanol, $\mathrm{I} 20 \mathrm{ml}$.

\section{RESULTS}

\section{Glutamine and the levels of glutamine synthetase}

We first describe experiments which show that the apparent rate of formation of glutamine synthetase was largely determined by the size of the cell pool of glutamine.

Changes in enzyme amount in response to changes in the level of ammonia in the medium. In all our experiments we have found that any reduction in ammonia concentration below $4 \mathrm{~mm}$ resulted in an increase in enzyme level. For example, when yeast was allowed to exhaust its medium of ammonia, the specific activities of glutamine synthetase and the NAD-specific glutamate dehydrogenase increased greatly (Fig. I). The yeast responded rapidly to this fall in ammonia concentration, before the medium was completely exhausted. Even a small alteration to the ammonia supply could produce a compensatory change in enzyme amount. In one experiment, carried out in a turbidostat, in which the ammonia excess in the medium was allowed to rise steadily over several hours from $\mathrm{I} \cdot 05 \mathrm{mM}$ to $\mathrm{r} \cdot 33 \mathrm{~mm}$, there was a fall in glutamine synthetase specific activity from $39 \mathrm{mu} / \mathrm{mg}$ protein to 3 I $\mathrm{mu} / \mathrm{mg}$ protein. These and similar experiments established that the amount of glutamine synthetase present was finely attuned to the availability of ammonia in the culture medium, but they did not allow us to determine whether it was ammonia itself, or a product of its assimilation, that was responsible for the modulation of enzyme amount.

Amount of enzyme in yeast growing with different sources of nitrogen. The specific activity of glutamine synthetase varied considerably when yeast was grown with a number of different compounds as sole sources of nitrogen (Table I). Glutamate-grown yeast contained about five times as much enzyme as did ammonia-grown yeast. This increase probably reflected a limited internal availability of ammonia rather than that glutamate itself was acting as a co-inducer of enzyme synthesis, since the specific activities of the enzyme in yeast growing with ammonia and with glutamate and ammonia were similar. Glutamine-grown yeast, in turn, contained only about one-fifth as much enzyme as did ammonia-grown yeast, a clear indication that it was not simply ammonia availability that was determining the level of glutamine synthetase. 


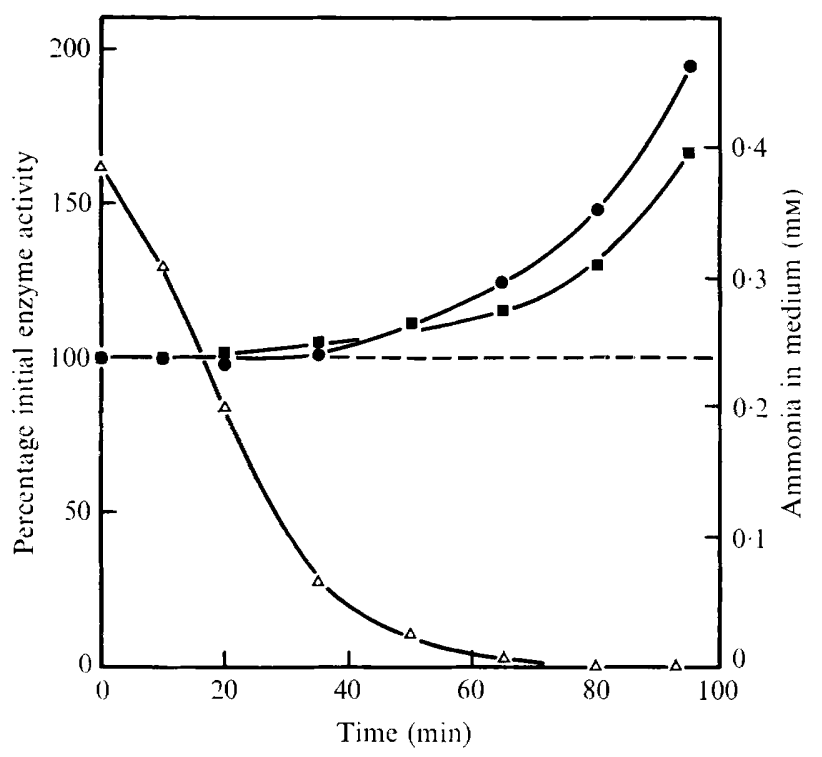

Fig. I. Changes in specific activity of glutamine synthetase and the glutamate dehydrogenases in yeast after a reduction in ammonia concentration in the medium. Yeast growing with excess ammonia (4 $\mathrm{mm}$ ) was filtered, washed, and resuspended in medium containing only low ammonia $(0.4 \mathrm{~mm})$. Enzyme specific activities are shown as percentages of those at the beginning of the experiment. $\triangle$, Ammonia in culture medium; glutamine synthetase $(100 \%=34.8 \mathrm{mu} / \mathrm{mg}$ protein); and $\boldsymbol{\square}$, NAD-specific glutamate dehydrogenase ( $100 \%=54.5 \mathrm{mu} / \mathrm{mg}$ protein). The dashed line shows that there is no change in the NADP-specific glutamate dehydrogenase (data from separate experiment; $100 \%=500 \mathrm{mu} / \mathrm{mg}$ protein).

Table I. Amounts of glutamine synthetase, NAD-specific glutamate dehydrogenase, and NADP-specific glutamate dehydrogenase in Candida utilis growing with different sources of nitrogen

Nitrogen source

Alanine

Ammonia

Ammonia and glutamate

Aspartate

Glutamate

Glutamine

Glycine

Nitrate

Urea
Glutamate dehydrogenases

Glutamine synthetase (mu/mg protein)

$\mathrm{I} 42$
29
33
$\mathrm{I} 57$
$\mathrm{I} 33$
6
77
38
63
(mu/mg protein)

\begin{tabular}{|c|c|c|}
\hline \multicolumn{2}{|c|}{$\begin{array}{l}\text { (mu/mg protein) } \\
\end{array}$} & \multirow{2}{*}{$\begin{array}{c}\text { Growth } \\
\text { rate } \\
\text { (generation } / \mathrm{h} \text { ) }\end{array}$} \\
\hline NAD-specific & NADP-specific & \\
\hline 232 & 535 & $0.44 \mathrm{I}$ \\
\hline 32 & 509 & 0.526 \\
\hline 34 & 448 & 0.455 \\
\hline 363 & 448 & 0.429 \\
\hline 162 & 494 & 0.500 \\
\hline 32 & 545 & 0.526 \\
\hline $4 I$ & 592 & 0.455 \\
\hline $3 I$ & 936 & 0.496 \\
\hline 40 & I I9 I & $0.44 \mathrm{I}$ \\
\hline
\end{tabular}

The initial nitrogen content of the medium was $20 \mathrm{~mm}$. Cultures were harvested in the exponential phase of growth for the preparation of extracts and the assay of enzyme activities. The values shown are the means of assays carried out on three different crude extracts prepared from the same culture. Glutamine synthetase activity was determined by the synthetase assay. 


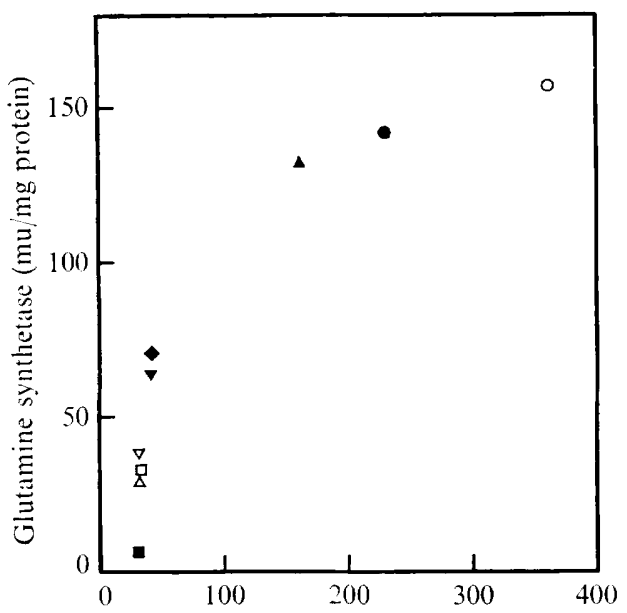

NAD-specific glutamate dehydrogenase

Fig. 2. Relationship between specific activity of glutamine synthetase and that of the NADspecific glutamate dehydrogenase in yeast growing with different sources of nitrogen., Alanine; $\triangle$, ammonia; $\square$, ammonia and glutamate; $O$, aspartate; $\boldsymbol{\Delta}$, glutamate; $\boldsymbol{\square}$, glutamine; glycine: - , nitrate; and $\boldsymbol{\nabla}$, urea.

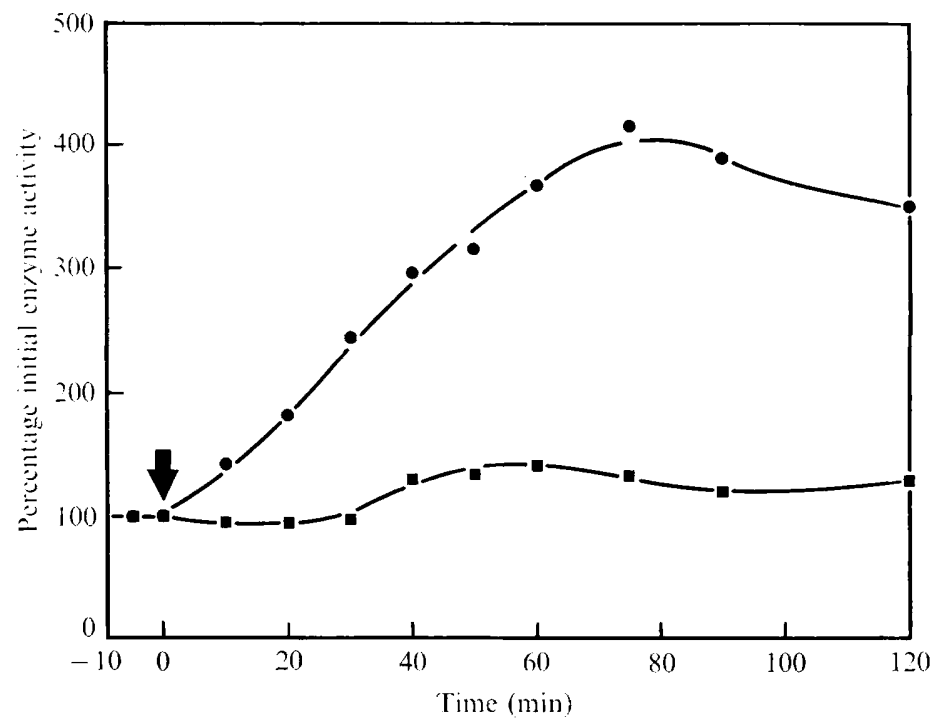

Fig. 3. Changes in specific activity of glutamine synthetase and the NAD-specific glutamate dehydrogenase after transfer of yeast from glutamine to ammonia. Exponentially growing yeast in medium containing glutamine $(6 \mathrm{~mm})$ was filtered, washed, and resuspended in medium containing ammonia $(6 \mathrm{~mm})$. Enzyme activities are shown as percentages of those at the beginning of the experiment. Glutamine synthetase $(100 \%=6.68 \mathrm{mu} / \mathrm{mg}$ protein); and $\mathbf{\square}$, NAD. specific glutamate dehydrogenase ( $100 \%=20 \cdot 8 \mathrm{mu} / \mathrm{mg}$ protein). 


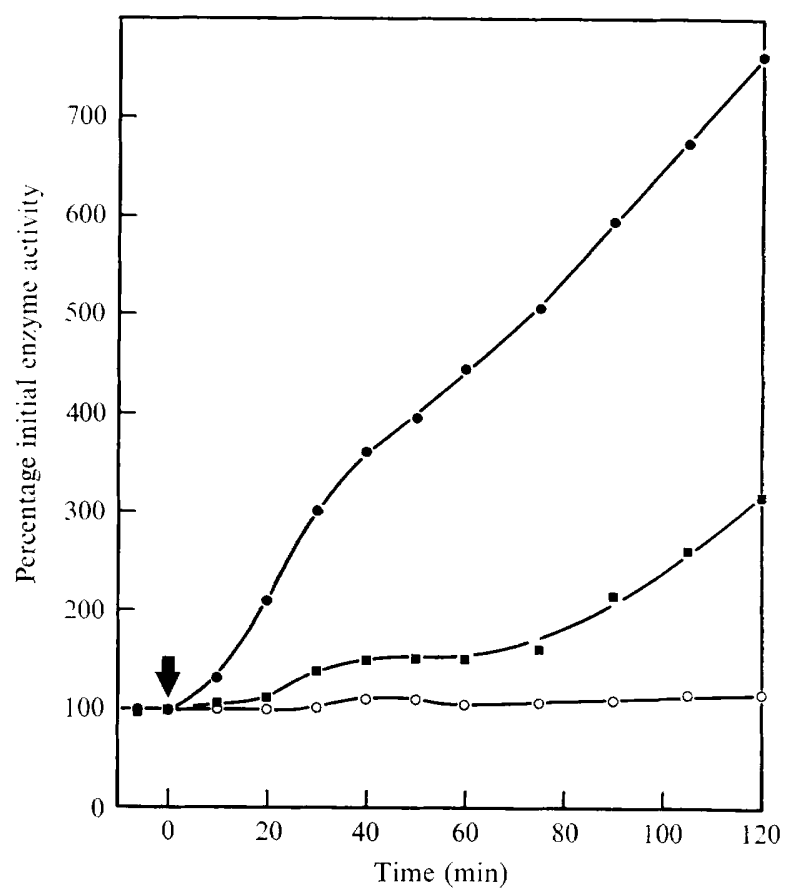

Fig. 4. Changes in specific activity of glutamine synthetase and the glutamate dehydrogenases after transfer of yeast from glutamine to glutamate. Exponentially growing yeast in medium containing glutamine $(6 \mathrm{~mm})$ was filtered, washed, and resuspended in medium containing glutamate $(6 \mathrm{~mm})$. Enzyme activities are shown as percentages of those at the beginning of the experiment. Glutamine synthetase (100\% $\%=8.8 \mathrm{mu} / \mathrm{mg}$ protein); $\boldsymbol{\square}$, NAD-specific glutamate dehydrogenase $(100 \%=2 \mathrm{r} \cdot 2 \mathrm{mu} / \mathrm{mg}$ protein); and $\mathrm{O}$, NADP-specific glutamate dehydrogenase $(100 \%=467 \mathrm{mu} / \mathrm{mg}$ protein $)$.

Although there was similar variation in the specific activity of the NAD-specific glutamate dehydrogenase, this enzyme and glutamine synthetase were not subject to parallel or coordinate regulation (Fig. 2). Thus yeast growing on some of the nitrogen sources differed considerably in its relative content of glutamine synthetase, but not in that of the NAD-specific glutamate dehydrogenase. The almost constant specific activity of this enzyme under different nutritional conditions may have meant that we were observing the fully repressed rate of enzyme synthesis. Yeast grown on alanine, aspartate, or glutamate contained very high amounts of the NAD-specific glutamate dehydrogenase, and it is probable that its functioning was here providing the ammonia required for amide synthesis and other reactions. Glutamine-grown yeast contained only very little of this enzyme activity, either because ammonia was being supplied through the action of a glutaminase, or, more likely, because glutamine was able to replace ammonia in many cell reactions. Glycinegrown and urea-grown yeast also contained low activities of the enzyme, and it is possible that here again ammonia requirements were being met by processes other than the deamination of glutamate. There was much less variation in the amount of the NADP-specific glutamate dehydrogenase: no obvious correlation existed between the amount of the NADP-specific glutamate dehydrogenase and that of either glutamine synthetase or the NAD-specific enzyme.

Changes in amount of enzyme after changes in nitrogen source. That ammonia does not act directly as a co-repressor of the formation of glutamine synthetase was confirmed by 


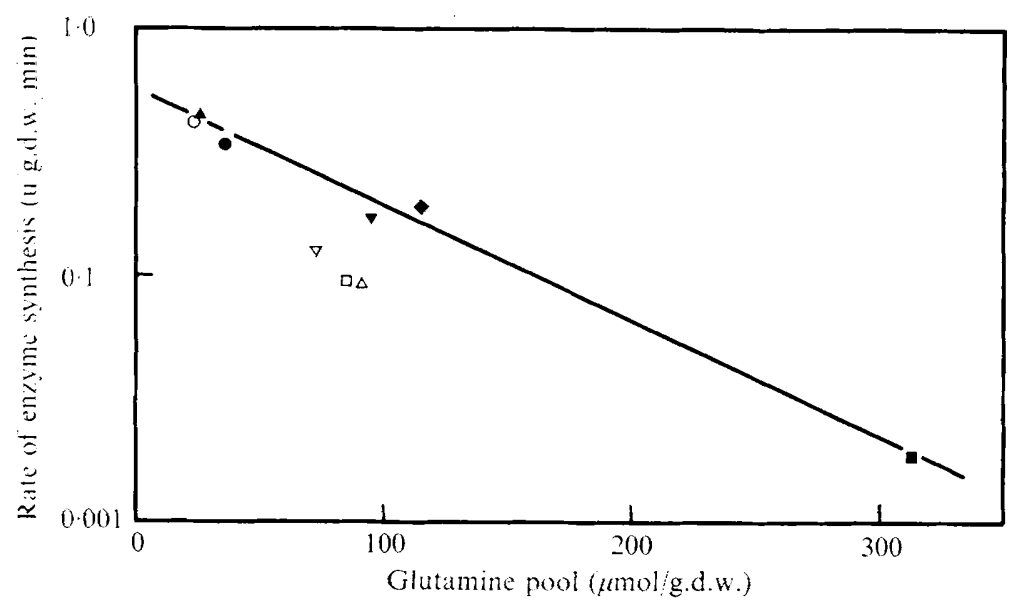

Fig. 5. Relationship between rate of glutamine synthetase formation and concentration of glutamine in the metabolic pool. Yeast was grown on a number of compounds each as sole source of nitrogen. The cells were harvested during the exponential phase of growth and measurements of the specific activity of glutamine synthetase, growth rate, and protein content of the cells were obtained to calculate the steady-state rate of enzyme synthesis. Samples were also analysed for their glutamine content. $\bigcirc$, Alanine; $\triangle$, ammonia; $\square$, ammonia and glutamate; $\bigcirc$, aspartate; $\boldsymbol{\Lambda}$, glutamate; $\boldsymbol{\square}$, glutamine; $\diamond$, glycine; $\nabla$, nitrate; and $\nabla$, urea. g.d.w. $=$ Grams dry weight.

an experiment in which glutamine-grown yeast was transferred to medium containing ammonia. There was an immediate and sustained rise in the specific activity of glutamine synthetase (Fig. 3) at the same time as there was a rise in the cell pool of ammonia and a rapid fall in that of glutamine. There was only a small increase in the NAD-specific glutamate dehydrogenase, another indication that the two enzymes are independently regulated. On transfer of glutamine-grown yeast to glutamate (Fig. 4), there was similarly a rapid increase in glutamine synthetase, this time accompanied by a delayed increase in the NAD-specific glutamate dehydrogenase. There was little change in the NADP-specific glutamate dehydrogenase.

\section{Glutamate and the rate of formation of glutamine synthetase}

The results so far described are consistent with the idea that glutamine itself is the co-repressor of enzyme synthesis. If this were so, then a simple inverse correlation might be expected between the rate of enzyme synthesis and the size of the glutamine pool.

It is possible to calculate the absolute rate of enzyme synthesis from the following expression:

$$
\frac{\mathrm{d} E}{\mathrm{~d} t}=\frac{\mathrm{d} x / \mathrm{d} t}{x} \times \frac{E}{x}+\frac{\mathrm{d} E / x}{\mathrm{~d} t}
$$

where $x$ is the mass of yeast/unit volume of culture, $t$ the time, and $E$ amount of enzyme/unit volume of culture. Because estimates of enzyme concentration, $E$, are normally based on measurements of enzyme activity, one must first establish that the activity per molecule of enzyme is constant under different conditions of synthesis. This is necessary because it has been found that the specific activity of glutamine synthetase (and certain other kinetic parameters) from Escherichia coli depends upon the extent to which it is adenylated (Shapiro \& Stadtman, 1970) and this is sensitive to the nutritional conditions that obtain 


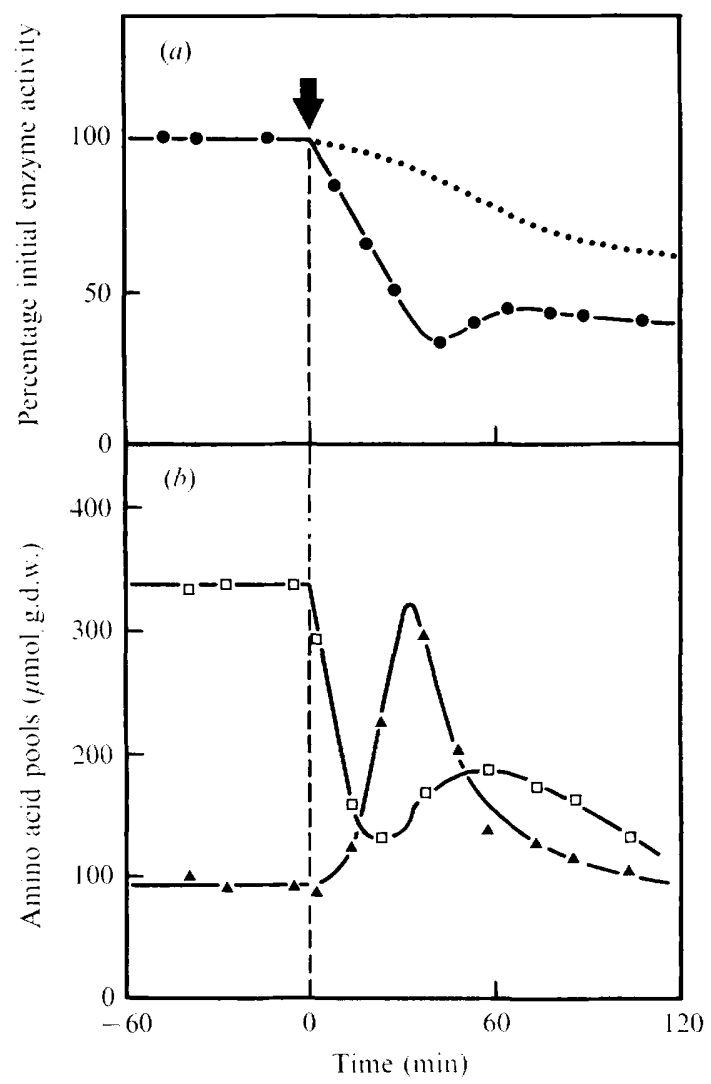

Fig. 6. Changes in (a) the specific activity of glutamine synthetase, and $(b)$ the pools of glutamate and glutamine after a change in nitrogen source from glutamate to ammonia. Yeast was grown in a turbidostat in medium containing a slight excess of glutamate (Io $\mu \mathrm{g} \mathrm{N} / \mathrm{ml}$ ). At the time shown, ammonia was added directly to the culture $\left(0.15 \mathrm{~g} \mathrm{NH}_{4} \mathrm{Cl} / \mathrm{I}\right)$, and the input medium was changed to one containing ammonia, 83.5 $\mu \mathrm{g} \mathrm{N} / \mathrm{ml}$. O, Glutamine synthetase ( $100 \%=140 \mathrm{mu} / \mathrm{mg}$ protein); ..., decrease of enzyme activity expected from repression and subsequent dilution by growth, assuming immediate cessation of enzyme synthesis; $\boldsymbol{\Lambda}$, glutamine pool; $\square$, glutamate pool.

when the enzyme is isolated. Moreover, it is also known that the yeast enzyme can exist in several active forms of differing molecular weight and properties (Sims, Toone \& Box, 1974). However, an examination of some of the 'indicator' kinetic parameters of enzyme originating from yeast grown on different sources of nitrogen (synthetase:transferase ratio, $\mathrm{pH}$ optimum, $K_{m}$ of the enzyme for glutamate and ammonia) suggested that we were dealing with an enzyme with identical properties in these studies. It has also been established that enzyme turnover in exponentially dividing yeast growing on glutamine is negligible (Ferguson \& Sims, 1974) and hence the application of the equation shown above is valid.

The results of these calculations are shown in Fig. 5 ; there was a good correlation between the rate of enzyme formation and the size of the glutamine pool but not that of the ammonia pool. Data obtained for yeast growing on nitrate, ammonia alone, or on ammonia plus glutamate fitted the correlation line less well. 

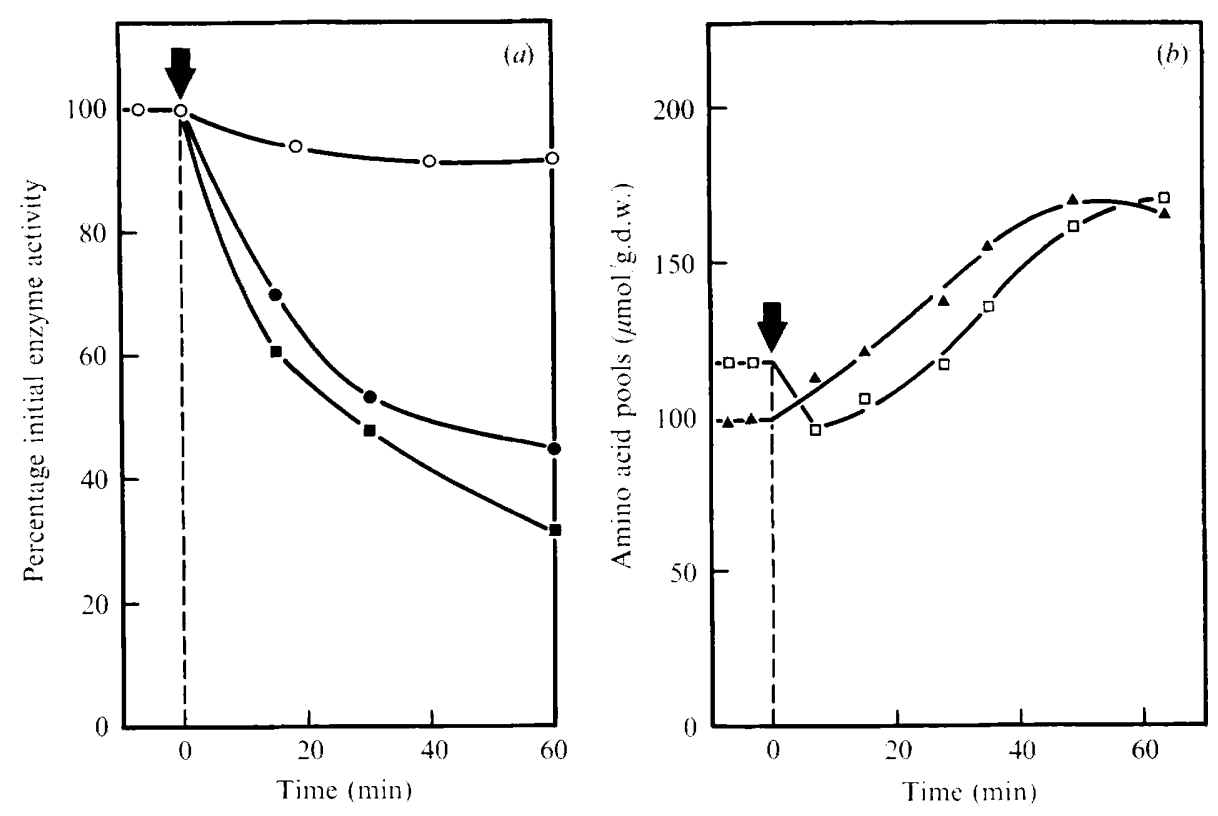

Fig. 7. (a) Changes in the specific activity of glutamine synthetase after the addition of ammonia or glutamine to yeast previously growing with glycine (glutamine synthetase, $100 \%=80 \mathrm{mu} / \mathrm{mg}$ protein). $\bigcirc$, Yeast transferred to new medium containing glycine $(6 \mathrm{~mm})$; 9 , ammonia $(6 \mathrm{~mm})$ added to medium; and $\boldsymbol{\square}$, glutamine $(6 \mathrm{~mm})$ added to medium. $(b)$ Changes in the soluble pools of glutamate $(\square)$ and glutamine $(\boldsymbol{\Delta})$ after the addition of ammonia to glycine-grown yeast.

\section{Glutamine and the inactivation of glutamine synthetase}

In earlier papers (Ferguson \& Sims, 197I; Sims \& Ferguson, 1974), we showed that in yeast the rapid and readily reversed inactivation of glutamine synthetase is an important mechanism by which regulation of glutamine synthesis can be achieved. We now describe some experiments examining the conditions under which such inactivation occurs.

Ammonia was added to a yeast culture growing in a turbidostat with glutamate as sole source of nitrogen, and at the same time the input medium was changed to one containing ammonia so that the nitrogen content of the culture medium was maintained almost constant during the transition. The addition of ammonia resulted in rapid inactivation of glutamine synthetase (Fig. 6a). This was accompanied by a threefold increase in the cell pool of glutamine and an equally dramatic reduction in the pool of glutamate, such that within $20 \mathrm{~min}$ it had fallen to less than half its original level (Fig. $6 b$ ). Later changes in the synthetase followed a distinctive oscillatory pattern, with specific activity rising and falling in a manner that closely paralleled fluctuations in the pool of glutamate. Only after one generation's growth did the amino acid pools and the specific activity of the synthetase approach the values characteristic of steady-state growth on ammonia.

However, inactivation of the synthetase was not observed only when yeast growing on glutamate was suddenly provided with ammonia or glutamine; it appeared to be a more general phenomenon. Thus it occurred when glutamine was added to yeast growing on aspartate or alanine, or when glutamine or ammonia was added to yeast previously grown on glycine as sole source of nitrogen (Fig. 7a). It also occurred when, in a turbidostat experiment, the supply of ammonia was restored to yeast that had been subjected to a 


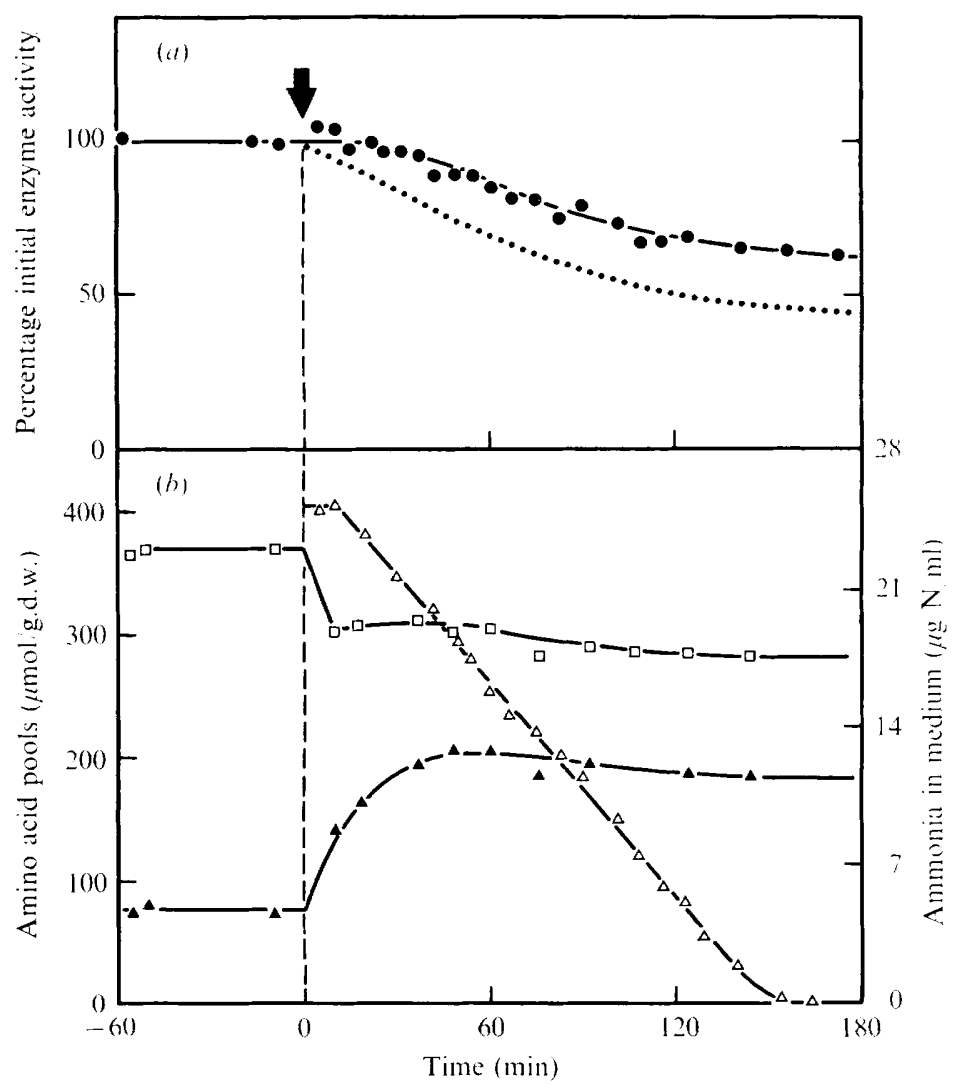

Fig. 8. Changes in (a) the specific activity of glutamine synthetase and $(b)$ the pools of glutamate and glutamine after the addition of a pulse of ammonia $\left(0.15 \mathrm{~g} \mathrm{NH}_{4} \mathrm{Cl}\right.$ to $\mathrm{I} \cdot 8 \mathrm{l}$ of culture) to yeast previously growing in a turbidostat with glutamate (15 $\mu \mathrm{g} \mathrm{N} / \mathrm{ml}$ excess) as sole nitrogen source. 0 , Glutamine synthetase ( $100 \%=135 \mathrm{mu} / \mathrm{mg}$ protein) $; \ldots$, decrease of enzyme activity expected from repression and subsequent dilution by growth, assuming immediate cessation of enzyme synthesis; $\boldsymbol{\Lambda}$, glutamine pool; $\square$, glutamate pool; $\triangle$, ammonia in medium.

period of nitrogen deprivation. During this time there had been an increase in amount of enzyme (Sims \& Ferguson, I974).

Although we have found enzyme inactivation usually to be associated with an increase in the cell concentration of glutamine, the association was not mandatory. Thus there could be an increase in the glutamine pool without inactivation occurring, and in other instances inactivation occurred even though there had been little or no change in the size of the glutamine pool. For example, when a pulse of ammonia was added to yeast maintained on glutamate, the enzyme was not inactivated but its synthesis was repressed (Fig. $8 a$ ). Measurement of the metabolite pools showed that whereas the pool of glutamine had risen appreciably there was only a slight fall in that of glutamate (Fig. $8 b$ ). On the other hand, when yeast grown on aspartate or glutamate was transferred to medium deficient in both carbon and nitrogen, the enzyme was rapidly inactivated (Fig. 9a). There was no accompanying increase in the glutamine pool, but inactivation could be correlated with a decrease in the soluble pool of glutamate (Fig. $9 b$ ). These observations confirmed the importance of glutamine in repressing the formation of the enzyme; they also suggested that enzyme inactivation 

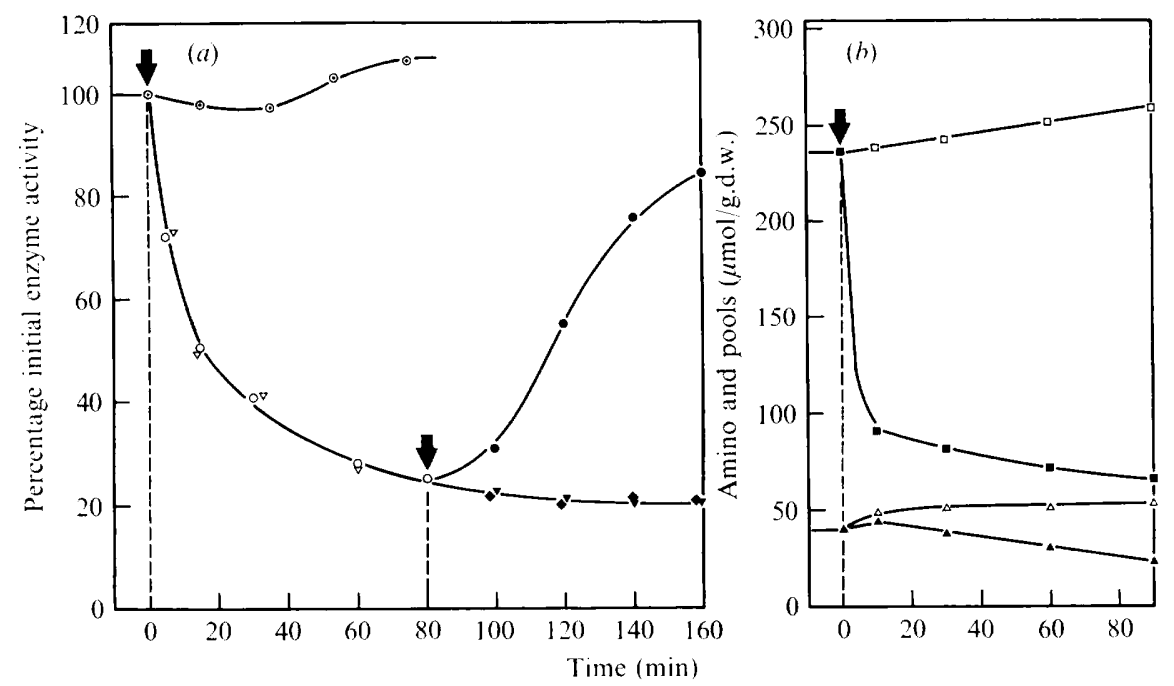

Fig. 9. (a) Changes in the specific activity of glutamine synthetase after the transfer of yeast from complete medium to medium lacking a carbon or nitrogen source. Yeast growing on glucose/glutamate medium (initially $6 \mathrm{~mm}$-glutamate) was filtered, washed, and transferred to: $\odot$, medium containing glucose but no nitrogen source; $\nabla$, medium containing glutamate but no glucose; $O$, medium containing no carbon or nitrogen source. After 80 min the culture in medium deficient in both carbon and nitrogen was divided into three parts and to these was added: glucose $(1 \circ \mathrm{g} / \mathrm{l}) ; \nabla$, glutamate $(3 \mathrm{~g} / \mathrm{l}) ; \diamond, \alpha$-oxoglutarate $(\mathrm{I} \cdot 5 \mathrm{~g} / \mathrm{l})$. (b) Changes in the soluble pools of glutamate and glutamine after the transfer of yeast from complete medium to medium containing glutamate $(3 \mathrm{~g} / \mathrm{l})$ but no glucose ( $\square$, glutamate; $\triangle$, glutamine) or to medium containing no nitrogen or carbon source ( $\mathbf{\square}$, glutamate; $\boldsymbol{\Delta}$, glutamine).

might occur as a consequence of a reduction in the pool of glutamate - owing to its diversion into glutamine - rather than of any build-up in glutamine itself. Stabilization of the enzyme by glutamate could also account for the relationship noted earlier (see Fig. 6) between oscillations in enzyme amount and oscillations in the glutamate pool. Subsequent experiments have shown, however, that this explanation is far too simple. The addition of ammonia to glycine-grown yeast resulted in an extensive build-up of the glutamine pool accompanied by an equally large, although somewhat delayed, rise in the glutamate pool (Fig. $7 b$ ), and yet inactivation of the synthetase was so rapid that within $40 \mathrm{~min}$ of the addition of ammonia, the specific activity of the enzyme had fallen to the level appropriate for growth on ammonia (Fig. 7a).

The process of inactivation is further complicated by the fact that it appears to involve more than changes in the relative concentrations of glutamate and glutamine. Thus, for example, when glutamate-grown yeast was transferred to medium containing glutamate but not glucose, the synthetase was rapidly inactivated (Fig. 9a) despite the fact that the pools of glutamate and glutamine remained virtually unchanged (Fig. $9 b$ ). Subsequent addition of glutamate or $\alpha$-oxoglutarate to yeast in which the enzyme had been inactivated had no effect, whereas addition of glucose resulted in the restoration of enzyme activity (Fig. 9a). Here, continued carbon metabolism seemed to be more important in determining the amount of enzyme present than did any changes in the relative concentrations of glutamate and glutamine. Other observations (not reported here in detail) confirmed that carbon metabolism was probably necessary for the maintenance of glutamine synthetase activity. It was found that although yeast grown with glucose and ammonia contained lower specific 
activities of the synthetase than did yeast growing in medium containing glycerol and glutamate (V. Box and A. P. Sims, unpublished), the transfer of yeast from glucose-ammonia to glycerol-glutamate medium brought about rapid inactivation of the enzyme. Only after about $30 \mathrm{~min}$ did the activity of the synthetase return to the levels characteristic of growth on glycerol and glutamate. It is possible that in this instance inactivation was a result of the inability of glucose-grown yeast to metabolize glycerol immediately after transfer and hence of its inability either to meet its energy requirements or to maintain the concentration of metabolite(s) responsible for inhibiting the inactivating system. However, continued exposure to the new carbon source would lead to the induction of the enzymes associated with glycerol assimilation, and hence to a restoration of enzyme activity.

\section{DISCUSSION}

In many micro-organisms, the amount of glutamine synthetase present varies with the nitrogen source provided for growth (Pateman, 1970; Shapiro \& Stadtman, 1970). Although it has been suggested that glutamate and some other amino acids, such as leucine and isoleucine, actually induce the formation of glutamine synthetase (Wu \& Yuan, I968), it is more usually considered that it is the availability of ammonia that determines the amount of enzyme present, and that ammonia itself acts as a co-repressor of enzyme synthesis (Kohlaw, Drägert \& Holzer, 1965). In most cases, however, it has not been possible to ascertain unequivocally whether it is ammonia, as such, that regulates the amounts of glutamine synthetase or whether it is glutamine, a product of ammonia assimilation, that is effective.

Our results establish that in Candida utilis it is glutamine, and not ammonia, that is a co-repressor of enzyme synthesis. A striking correlation was demonstrated between specific activity of glutamine synthetase and size of glutamine pool. There was no such correlation between enzyme amount and the ammonia pool, even though the concentration of glutamine might be expected to be an indication of the availability of ammonia.

Glutamine, as well as governing the rate at which glutamine synthetase is formed, may, under certain conditions, be directly or indirectly responsible for initiating the inactivation of the enzyme. The experiments described indicate that the inactivation process is normally associated with conditions that produce marked and sudden changes in the pools of metabolic intermediates. Although these physiological experiments have not enabled us to determine unequivocally whether one or several effector molecules are responsible for promoting inactivation or whether glutamine overproduction or glutamate depletion is involved, it does seem likely that changes in the relative concentrations of these two amino acids may either reflect or impose changes on the flux of another intermediate(s) which acts more directly. A better understanding of the different controls involved will have to await the study in vitro of the inactivating system.

The amount of the NAD-specific glutamate dehydrogenase also varies with the nitrogen source supplied. Under some growth conditions, the functioning of this enzyme probably provides the ammonia required for amide synthesis (Hierholzer \& Holzer, 1963) and it might therefore be expected that its specific activity and that of glutamine synthetase would respond in a similar manner to the availability of ammonia. Although Kohlaw et al. (1965) have claimed that the two enzymes are subject to parallel regulation, our results have shown that they can be independently regulated.

Glutamine has been recognized as playing two distinctive roles in the regulation of glutamine biosynthesis in micro-organisms: of controlling the formation of glutamine synthetase, and of controlling the activity of the enzyme. Thus in Lactobacillus arabinosus 
it acts as a co-repressor of the formation of the enzyme (Ravel, Humphreys \& Shive, 1965). In Bacillus subtilis (Deuel \& Stadtman, 1970) glutamine synthetase is directly inhibited by glutamine. In Escherichia coli, the adenylation and de-adenylation of glutamine synthetase, and hence modulation of the activity of the enzyme and of its susceptibility to feedback inhibition, is in response to variation in the relative cellular concentrations of glutamine and $\alpha$-oxoglutarate (Shapiro \& Stadtman, I970; Holzer \& Duntze, I97I). In Candida utilis there appear to be no feed-back controls, either direct or indirect, imposed on the pathway of glutamine synthesis (Sims \& Ferguson, 1972; 1974). There is thus a real need for very precise control of enzyme synthesis, and this seems to have been met by the evolution of a system in which even small changes in the glutamine pool can modulate both the amount of enzyme and its rate of synthesis.

This work was supported by the Science Research Council by grant B/SR/5II6. The authors thank Mrs J. Toone for her skilled technical assistance and S. Howitt for some amino acid analyses. We also thank E. G. Bollard for reading the manuscript.

\section{REFERENCES}

Deuel, T. F. \& Stadtman, E. R. (1970). Some kinetic properties of Bacillus subtilis glutamine synthetase. Journal of Biological Chemistry 245, 5206-5213.

Ferguson, A. R. \& Sims, A. P. (I97I). Inactivation in vivo of glutamine synthetase and NAD-specific glutamate dehydrogenase: its role in the regulation of glutamine synthesis in yeasts. Journal of General Microbiology 69, 423-427.

Fergusor, A. R. \& Sims. A. P. (I974). The regulation of glutamine metabolism in Candida utilis: the inactivation glutamine synthetase Journal of General Microbiology 8o, 173-185.

Hierholzer, G. \& Holzer, H. (1963). Repression der Synthese von DPN-abhängiger Glutaminsäuredehydrogenase in Saccharomyces cerevisiae durch Ammoniumionen. Biochemische Zeitschrift 339, $175-185$.

Hirs, G. H. W., Moore, S. \& Stein, W. H. (1954). The chromatography of amino acids on ion exchange resin. Use of volatile buffers for elution. Journal of the American Chemical Society 76, 6063-6065.

Holzer, H. \& DUNTze, W. (1971). Metabolic regulation by chemical modification of enzymes. Annual Revien of Biochemistry 40, 345-374.

Kohlaw, G., DräGert, W. \& Holzer, H. (I965). Parallel-Repression der Synthese von Glutamin-Synthetase und DPN-abhängiger Glutamat-Dehydrogenase in Hefe. Biochemische Zeitschrift 34I, 224-238.

Pateman, J. A. (1970). Regulation of synthesis of glutamate dehydrogenase and glutamine synthetase in micro-organisms. Biochemical Journal I15, 769-775.

Ravel, J. M., Humphreys, J. S. \& Shive, W. (1965). Control of glutamine synthesis in Lactobacillus arabinosus. Archives of Biochemistry and Biophysics III, 720-726.

Shapiro, B. M. \& Stadtman, E. R. (1970). The regulation of glutamine synthesis in microorganisms. Annual Review of Microbiology 24, 50I-524.

Sims, A. P. \& Ferguson, A. R. (1972). The role of enzyme inactivation in the regulation of glutamine synthesis in yeast: in vivo studies using ${ }^{15} \mathrm{~N}$. In 6 . Wissenschaftliche Konferenz der Gesellschaft Deutscher Naturforscher und Ärzte, Second International Symposium on Metabolic Interconversion of Enzymes, Rottach-Egern 1971, pp. 26r-276. Edited by O. Wieland, E. Helmreich and H. Holzer. Berlin, Heidelberg, New York: Springer Verlag.

Sims, A. P. \& Ferguson, A. R. (1974). The regulation of glutamine metabolism in Candida utilis: studies with ${ }^{15} \mathrm{NH}_{3}$ to measure in vivo rates of glutamine synthesis. Journal of General Microbiology 8o, I43-I 58.

Sims, A. P.. Toone, J. \& Box, V. (1974). The regulation of glutamine synthesis in the food yeast Candida utilis: the purification and subunit structure of glutamine sythetase and aspects of enzyme deactivations. Journal of General Microbiology 80 (in the Press).

Weatherburn, M. W. (1967). Phenol-hypochlorite reaction for determination of ammonia. Analytical Chemistry 39, 97 I-974.

Wu, C. \& YUAN, L. H. (1968). Regulation of synthesis of glutamine synthetase in Escherichia coli. Journal of General Microbiology 51, 57-65.

Yemm, E. W. \& Cocking, E. C. (1955). The determination of amino acids with ninhydrin. Analyst 8o, 209-2I4. 